\title{
On the role of polar molecules and the barrier for charge injection in OLEDs
}

\author{
S. Altazin ${ }^{1}$, S. Züfle ${ }^{1,2}$, E. Knapp², C. Kirsch², T. D. Schmidt ${ }^{3}$, L. Jäger ${ }^{3}$, \\ W. Brütting 3 , B. Ruhstaller ${ }^{1,2}$ \\ ${ }^{1}$ Fluxim AG, Winterthur, Switzerland \\ ${ }^{2}$ Zurich University of Applied Sciences, Institute of Computational Physics, Winterthur, \\ Switzerland \\ ${ }^{3}$ Experimental Physics IV, Institute of Physics, University of Augsburg, Germany
}

\begin{abstract}
Many electron transport layer materials (ETL) employed in state of the art organic light emitting diodes (OLEDs) are known to be polar. We combine for the first time simulations and electrical characterization of OLEDs based on polar ETL, in order to understand the impact of such materials on the device operation. Depending on the orientation of the dipole orientation, simulations predict either a benefit or a disadvantage of the polar ETL for the device performance. We also show that OLEDs featuring a polar material are perfectly suited for extracting mobility activation energy and Injection barrier from the anode to the ETL.
\end{abstract}

Keywords: Polar materials, OLEDs, simulation, characterization, injection barrier. 


\section{INTRODUCTION}

OLED technology is gaining ground as next generation device for display and lighting applications. For mobile devices in display and lighting, the power efficiency is one of the most important parameters. Several paths are followed in order to increase the efficiency of OLEDs: both optical (like light scattering for lighting applications [1]) and electrical for example using highly conductive hole transport layers (HTLs) and electron transport layers (ETLs) in order to decrease the operation voltage of the device. ETL materials often show a non-zero permanent molecular dipole moment [2]. When deposited in a thin film stack configuration, this effectively leads to a layer with a positive sheet charge density on one side and a negative one on the other (see figure 1). So far, only experimental studies have been performed to investigate the impact of the polarity on the device operation, and only compact models based on equivalent circuits have been proposed to simulate such devices [2,3]. For the first time we show that the well-established drift-diffusion formalism implemented in SETFOS 4.3 [4] is able to cope with these materials leading to quantitative simulations of OLEDs embedding a polar ETL material. This allows us to both simulate the specific behavior introduced by such polar materials but also to give directions to improve the device operation. In this contribution we will mainly study a traditional bi-layer OLED as shown in figure 1, but similar effects and conclusions can be found for more complex dyedoped multilayer devices [5]. In this contribution we make an extensive use of impedance spectroscopy characterization and modelling. Indeed this technique is known to be highly sensitive to the charges in the device; therefore this characterization technique is well suited to study the effect of polar materials on the device operation.

In section 2 we will introduce the basics of impedance spectroscopy as well as the basics of the mathematical model. In the second part, we will compare the simulation results with experimental characterization of OLEDs embedding a polar ETL material. This allows us to benchmark our approach and to extract some device and materials parameters. Finally, in a last part we will use our model to simulate the potential benefit of the ETL polarity on the IV curve of the device.
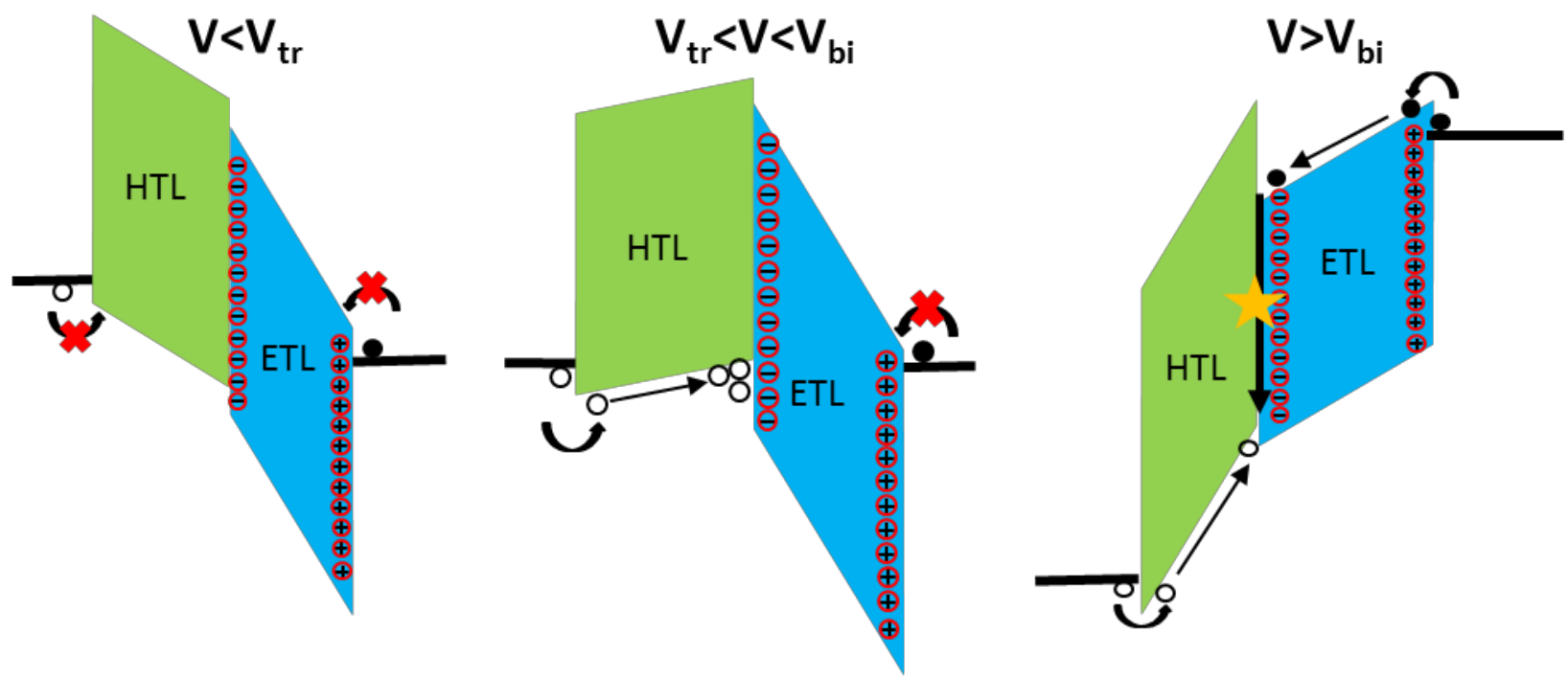

FIG. 1. Schematic band diagram of the bi-layer OLED used in this study. The ETL (Alq3) layer is supposed to have a molecular polar moment that is not randomly oriented, resulting in sheet charge densities on either side of the layer. This sheet charge density modifies the band diagram. 


\section{BASICS OF CHARACTERIZATION AND SIMULATIONS OF IMPEDANCE SPECTROSCOPY}

Impedance spectroscopy is a well-known technique to study OLED device operation. It has been extensively used to characterize the doping concentration [6] or the mobility [7,8] in organic and inorganic materials, as well as self-heating [9].

In this technique, the device is biased at a constant voltage $V_{0}$, on top of which small sinusoidal voltage $\left(V_{1}\right)$ is applied. The total voltage $(\mathrm{V})$ applied to the device is then:

$V=V_{0}+V_{1} \cdot \sin (2 \pi f t)$.

Here $f$ represents the frequency and the time. The basic concept of impedance spectroscopy is that the voltage $V_{1}$ is small enough such that the device behaves in a linear regime. Therefore the admittance of the device can be written as:

$\mathrm{Y}=\mathrm{J}_{\mathrm{ac}} / \mathrm{V}_{\mathrm{ac}}=\mathrm{G}+\mathrm{i} \omega \mathrm{C}$.

Where $\mathrm{J}_{\mathrm{ac}}$ represents the current flowing out of the device minus the steady state current and $\mathrm{V}_{\mathrm{ac}}=V_{1}$. $\exp (i 2 \pi f t)$

Finally, an equivalent capacitance $\mathrm{C}$ and conductance $\mathrm{G}$ of the device can be calculated. By varying the frequency $\mathrm{f}$, the capacitance versus frequency of the device can be measured, and by varying the constant voltage $\mathrm{V}_{0}$ one can measure the well-known C-V response of the device.

In order to simulate the device operation we use the well-established physical drift-diffusion formalism implemented in SETFOS 4.3. It consists of the continuity equation for electrons and holes coupled with the Poisson equation:

$\operatorname{div}\left(-\mu_{n} \cdot \boldsymbol{n} \cdot \vec{E}-\frac{k_{b} T}{q} \mu_{n} \cdot \overrightarrow{g r a d}(\boldsymbol{n})\right)-R=-\frac{\partial \boldsymbol{n}}{\partial t}$

$\operatorname{div}\left(\mu_{p} \cdot \boldsymbol{p} \cdot \vec{E}-\frac{k_{b} T}{q} \mu_{p} \cdot \overrightarrow{\operatorname{grad}}(\boldsymbol{p})\right)-R=-\frac{\partial \boldsymbol{p}}{\partial t}$

$\Delta \mathbf{V}=-\frac{q}{\varepsilon}(\boldsymbol{p}-\boldsymbol{n}+Q)$.

Where $\mu_{\mathrm{n}, \mathrm{p}}$ represents the mobility of electrons (n) and holes (p), respectively, $\mathrm{V}$ the electrostatic potential, $\mathrm{R}$ the Langevin recombination rate and $\mathrm{Q}$ the electrostatic fixed charge. This latter quantity is of primary importance for the simulation of polar materials: as explained previously, the non-zero permanent dipole moment of the molecules associated with a preferred orientation during the deposition process effectively leads to a layer with a positive sheet charge density on one side and a negative one on the other side. In our simulations, these charged layers are modeled by introducing a fixed charge density in the Poisson equation.

Finally in order to simulate impedance spectroscopy, the three variables: $\mathbf{n}, \mathbf{p}$ and $\mathbf{V}$ are replaced by [10]:

$n=n_{e q}+\boldsymbol{n}_{\boldsymbol{a c}} \cdot e^{i 2 \pi f t}$

$p=p_{e q}+\boldsymbol{p}_{\boldsymbol{a c}} \cdot e^{i 2 \pi f t}$

$V=V_{e q}+\boldsymbol{V}_{\boldsymbol{a c}} \cdot e^{i 2 \pi f t}$

Where the subscript eq denotes the equilibrium solution in steady state and the subscript ac denotes the harmonic solution. Then, assuming, that the variables $\mathrm{n}_{\mathrm{ac}}$, $\mathrm{p}_{\mathrm{ac}}$ and $\mathrm{V}_{\mathrm{ac}}$ are small enough (which is the basic assumption of impedance spectroscopy), the system is linearized and solved. The quantity $\mathrm{J}_{\mathrm{ac}}$, which represents the harmonic current flowing out of the device, can then be calculated as well as the equivalent admittance of the device. The approach described above has been implemented in the software SETFOS 4.3 [3] and allows for fast and reliable impedance spectroscopy simulations without having to solve the equations in a time domain regime which would be much more time consuming. 


\section{VALIDATION OF THE MODELLING APPROACH}

\subsection{Capacitance-voltage characteristic}

The Capacitance-voltage experiment $(\mathrm{C}-\mathrm{V})$ is known to be sensitive to the charge distribution in the device. Previous studies of Brütting et al. [2] have shown that the polar nature of Tris(8-hydroxyquinolinato) aluminium ( Alq $_{3}$ ) induces a typical signature in the $\mathrm{C}-\mathrm{V}$ experiment. Indeed, as in [2], and in figure 2 (left) two plateaus can be observed in the $\mathrm{C}-\mathrm{V}$ plot. Interestingly, the transition voltage $\left(\mathrm{V}_{\mathrm{tr}}\right)$, at which the capacitance changes, is directly related to the thickness of the $\mathrm{Alq}_{3}$ layer. This transition voltage occurs at negative values of the applied bias, suggesting the presence of mobile charges in the device for voltages below the $\mathrm{V}_{\mathrm{bi}}$ (built in voltage $\approx 2 \mathrm{~V}$ ). We find that $\mathrm{C}-\mathrm{V}$ simulations of such devices, using the approach developed in the previous section, can nicely reproduce this experimental observation (figure 2 right). In order to simulate the polar nature of the $\mathrm{Alq}_{3}$, a negative charge sheet density of $\sigma=1.2 \mathrm{mC} / \mathrm{m}^{2}$ was introduced at the interface between $\mathrm{Alq}_{3}$ and TPD and a positive one at the interface with the Calcium electrode, this value is in nice agreement with the one found in [11] using a Kelvin probe measurement.

In order to be sure that the steps in the $\mathrm{C}-\mathrm{V}$ measurements and simulations (capacitance increase below the turn-on voltage) were a consequence of the polarity of the material, we have run the same simulations (not shown here) assuming, this time, no fixed charges in the device $(\mathrm{Q}=0)$. As expected, the simulated $\mathrm{C}-\mathrm{V}$ curves did not show any kinks for negative voltages.
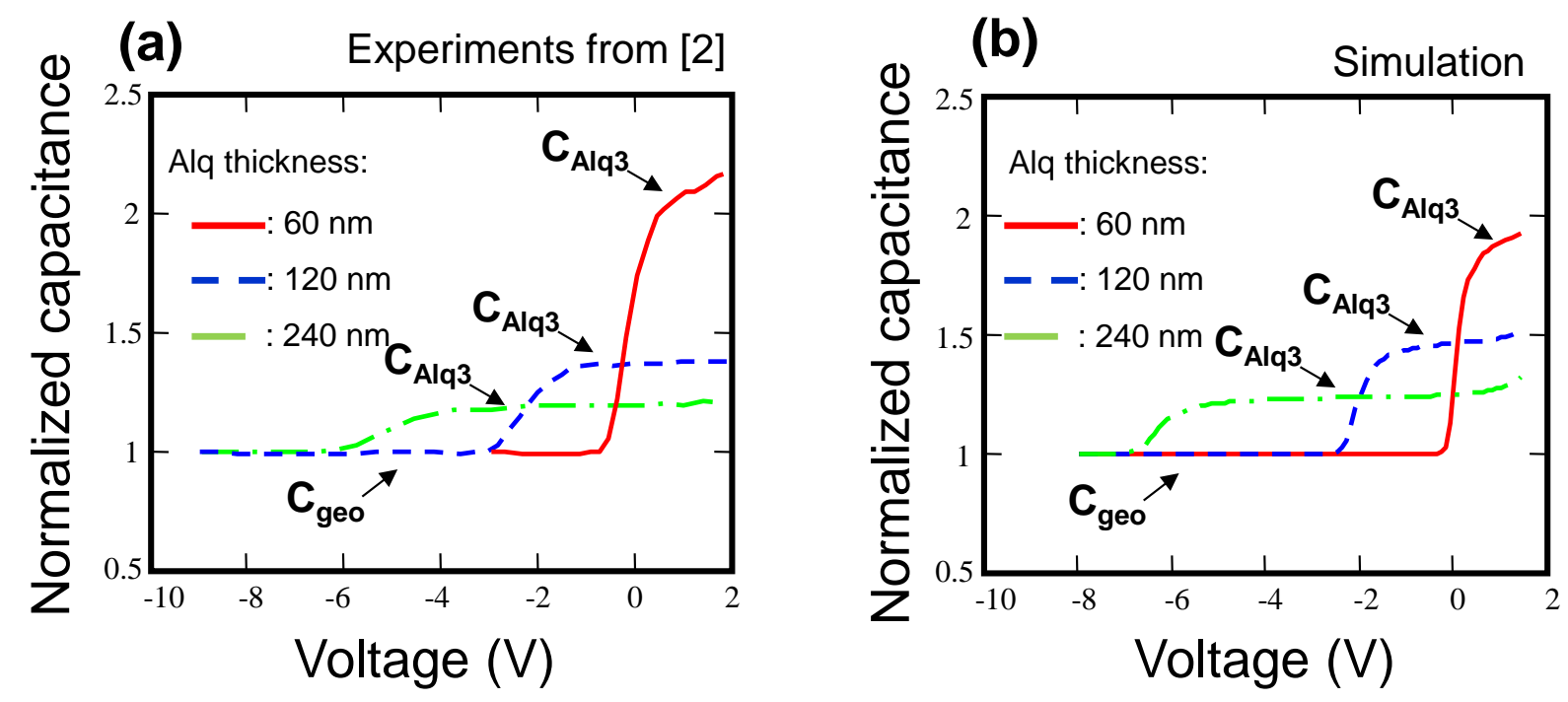

FIG. 2. Measured (left) from [2] and simulated (right) capacitance-voltage curves for OLEDs with different Alq3 layer thickness

In order to understand and explain the different regimes observed in the $\mathrm{C}-\mathrm{V}$ curve we have simulated the hole and electron concentration in the device in three different regimes (Figure 3) namely for $\mathrm{V}<\mathrm{V}_{\text {tr }}$ (red), for $\mathrm{V}_{\mathrm{tr}}<\mathrm{V}<\mathrm{V}_{\mathrm{bi}}$ (green) and for $\mathrm{V}>\mathrm{V}_{\mathrm{bi}}$ (green). In the following, we chose to simulate a device with a thick ETL (240nm) and with an HTL of 60 $\mathrm{nm}$.

One can see that for $\mathrm{V}=-8 \mathrm{~V}\left(\mathrm{~V}<\mathrm{V}_{\mathrm{tr}}\right)$ no holes nor electrons are present in the device, the OLED behaves as a perfect insulator, the capacitance that is simulated and characterized is therefore equal to the geometrical capacitance of the full OLED

$C_{g e o}=\frac{\varepsilon_{E T L} \cdot \varepsilon_{H T L}}{\varepsilon_{E T L} \cdot t_{H T L}+\varepsilon_{H T L} \cdot t_{E T L}}$ 
(a)

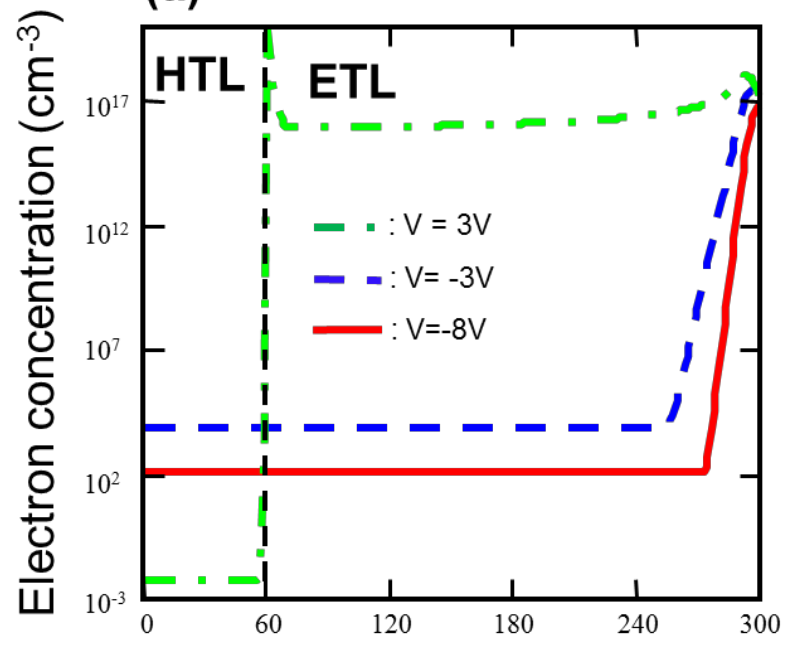

Distance from anode $(\mathrm{nm})$ (b)

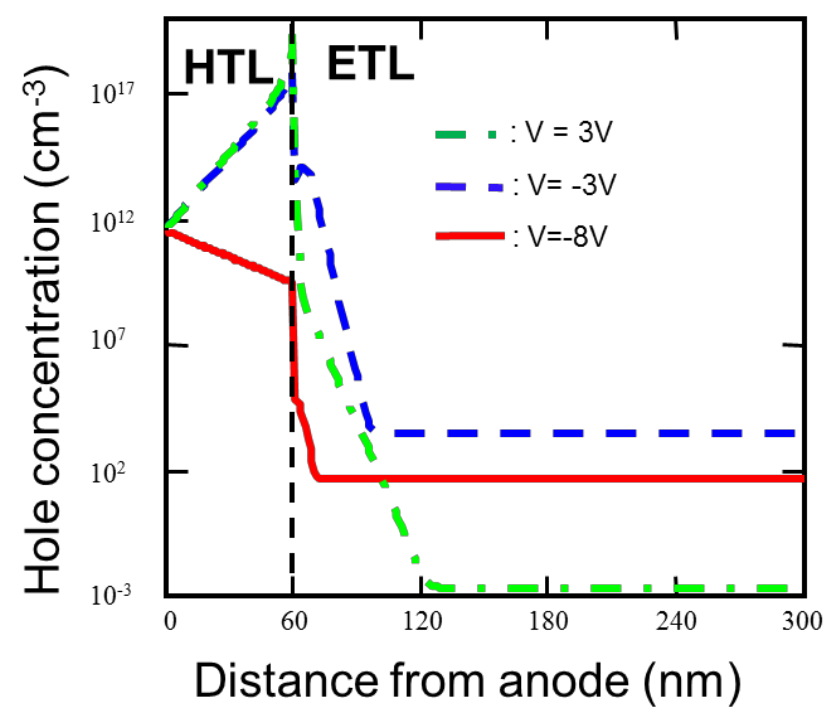



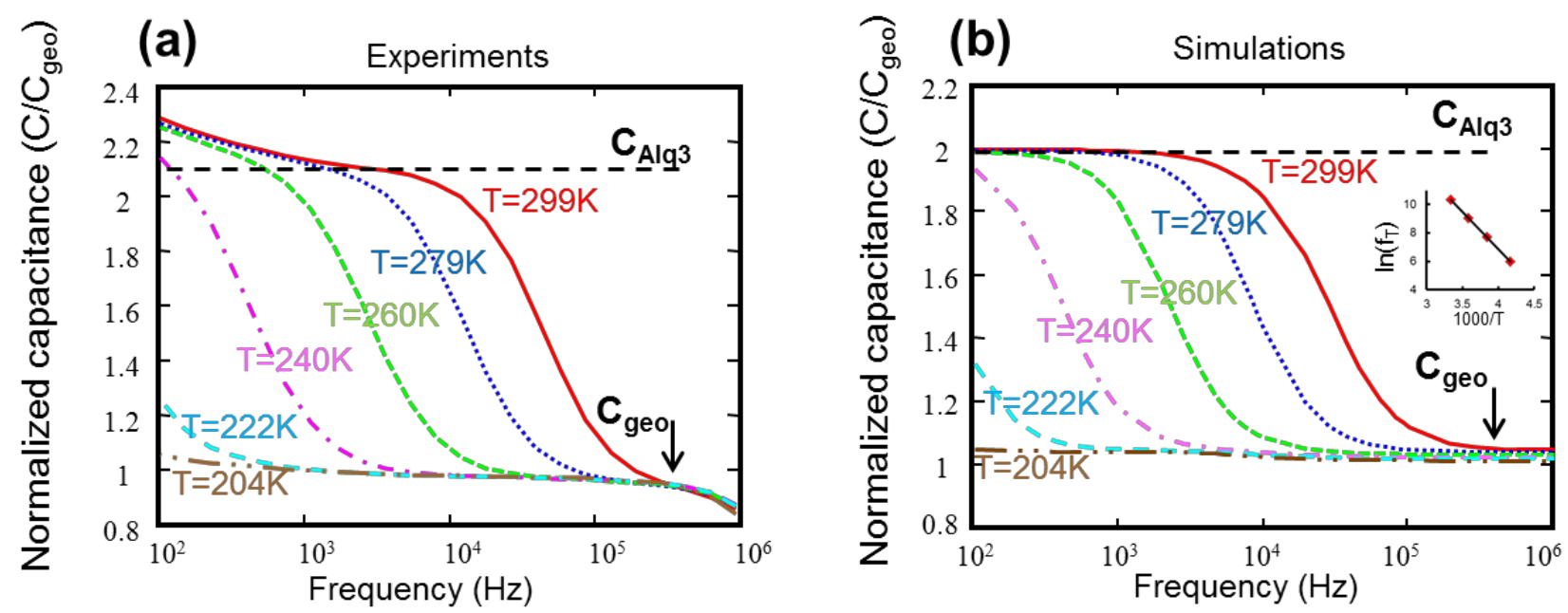
that the energy barriers holes have to overcome to jump from the anode to the HTL is $\Delta \mathrm{E}_{\text {inj }}=\Delta \mathrm{E}_{\mathrm{CFT}}-\Delta \mathrm{E}_{\mathrm{CELIV}}$, where $\Delta \mathrm{E}_{\text {inj }}$ represents the injection barrier, $\Delta \mathrm{E}_{\mathrm{CFT}}$ the activation energy extracted from the capacitance frequency experiment

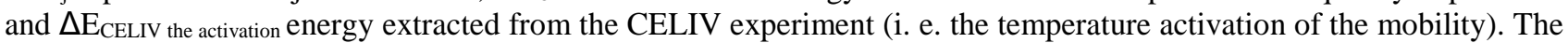
injection barrier was finally found equal to $0.25 \mathrm{eV}$.

(a)

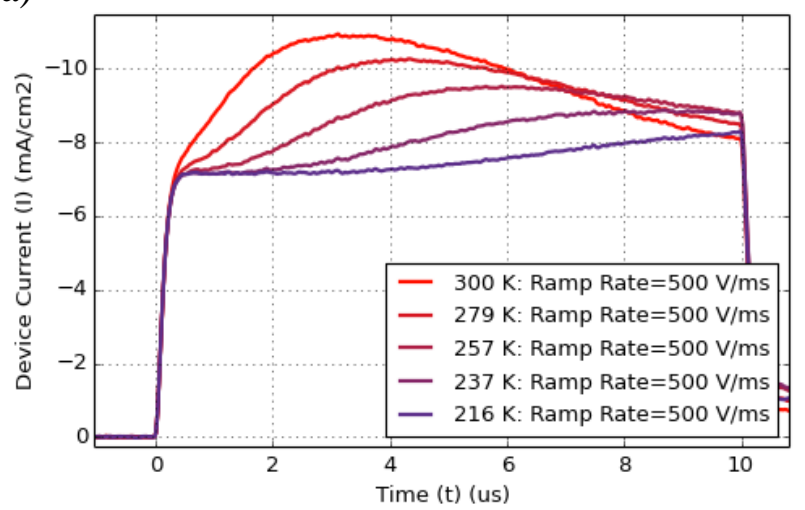

(b)

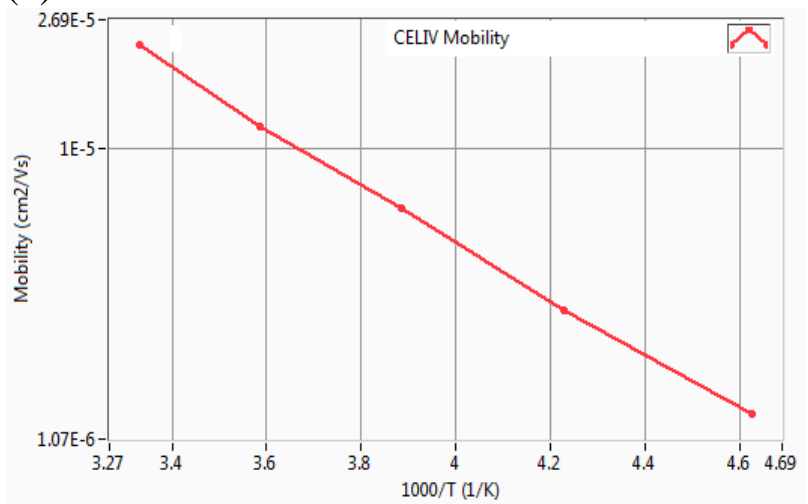

Fig. 5: CELIV measurements of a bilayer OLED as shown in figure 1 with a pre-bias voltage of $0 \mathrm{~V}$ (a) and extracted mobility from the time position of the maximum of the CELIV signal.

\section{IMPACT OF THE POLARITY ON THE IV CURVE}

In figure 6 we show the simulated IV curve of a bilayer (ITO/PEDOT(30nm)/ $N, N^{\prime}$-Di(1-naphthyl)- $N, N^{\prime}$-diphenyl-(1,1'biphenyl)-4,4'-diamine (NPB) $\left.(70 \mathrm{~nm}) / \mathrm{Alq}_{3}(40 \mathrm{~nm}) / \mathrm{Ca}(15 \mathrm{~nm}) / \mathrm{Al}\right)$ OLED device for three different cases: assuming a non-polar ETL, with a polar $\mathrm{Alq}_{3}$ and finally assuming a reversed polarity of the ETL (i. e. with a positive sheet charge density at the HTL/ETL interface). We can notice the increase of the current when the polar moment of the ETL is directed toward the NPB layer and a decrease of the current when the dipolar moment is inverted. This latter point has been experimentally observed by Noguchi et al.[15], where the orientation of the molecular dipoles of the ETL could be inverted using an alternative electron transport material: tris(7-propyl-8-hydroxyquinolinato) aluminum ( $\mathrm{Al}(7-p y q))$. The sheet charge densities found in [13] (1.1 mC. $\mathrm{m}^{-2}$ for the $\mathrm{Alq}_{3}$ and $3.1 \mathrm{mC} \cdot \mathrm{m}^{-2}$ for the 'inverted' ETL) were used for the simulations shown in figure 5. From these simulations we can conclude that introducing polar ETL can be beneficial for the OLED as the current can be increased leading to a decrease of the driving voltage and therefore an increase in power efficiency.

In order to understand the reason why the current is modified by using a polar ETL material, we have simulated the electron concentration inside the ETL layer (figure 5 right). We observe that when a negative sheet charge density is located at the HTL/ETL interface (and consequently with a positive sheet charge density at the cathode/ETL interface), the concentration of electrons is increased at the cathode interface, leading to an enhanced injection inside the ETL and thus increasing the current. When the polarity is inverted, the electron concentration is decreased leading to a reduced conductivity of the ETL reducing the current flowing through the device. These findings are in agreement with the work from Kinjo et al.[16] where they studied the Band diagram of $\mathrm{ALq}_{3}$ and $\mathrm{Al}(7-\mathrm{pyq})$ using photoelectron spectroscopy and photoelectron yield spectroscopy. 
(a)

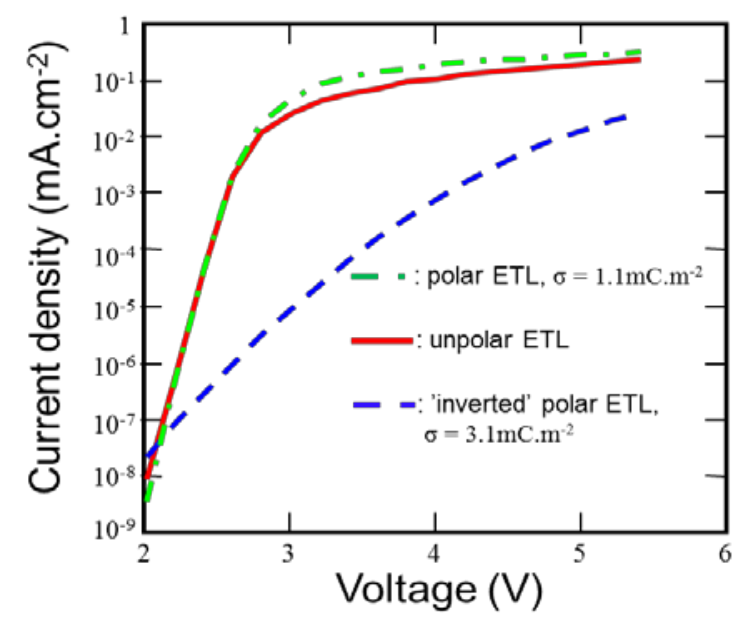

(b)

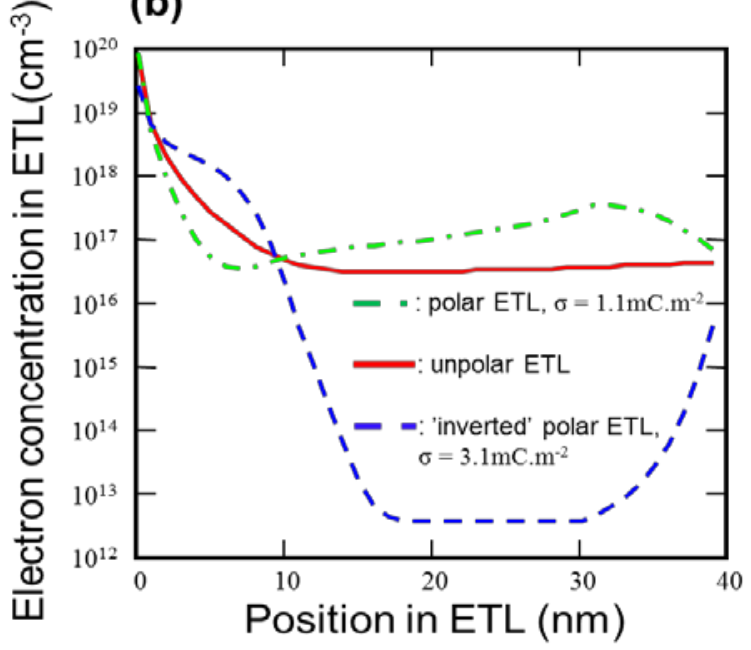

Fig.6: Simulated impact of the ETL polarity orientation on the IV curve of the OLED, and corresponding electron concentration in the

ETL for an applied voltage of 3V.

\section{CONCLUSION}

In this contribution we have shown that polar materials can be simulated using the commercially available simulation software SETFOS 4.3 introducing charge sheets on both sides of the polar layer. The comparison of measured and simulated capacitance as a function of bias, frequency and temperature allows quantifying the polarity of the ETL material. In a second step, the temperature activation energy observed in the temperature dependent capacitancefrequency characterization was investigated. Combining temperature dependent CELIV and temperature dependent capacitance-frequency measurements (both available with PAIOS 2.2) we were able to distinguish which part of the activation energy has to be attributed to the injection barrier and to the mobility activation energy. Finally we have shown that the polarity of the ETL can be beneficial if correctly oriented in the device as it can enhance the electron injection in the $\mathrm{Alq}_{3}$ layer. 


\section{REFERENCES}

[1] Altazin, S., Reynaud, C., Mayer, U. M., Lanz, T., Lapagna, K., Knaack, R., Penninck, L., Kirsch, C., Pernstich, K. P., Harkema, S., Hermes, D., and Ruhstaller, B., " Simulations, measurements, and optimization of OLEDs with a scattering layer” SID Int. Symp. Digest Tech. Papers 46(1), 564-567 (2015).

[2] Brütting, W., Berleb, S., Mückl, A. G., "Device physics of organic light-emitting diodes based on molecular materials”Org. Elec., 2 (1), 1-36 (2001).

[3] S. Nowy, W. Ren, A. Elschner, W. Lövenich, and W. Brütting, , "Impedance spectroscopy as a probe for the degradation of organic light-emitting diodes”, J. Appl. Phys., 107, 054501 (2010)

[4] Simulation software SETFOS version 4.3 by Fluxim AG, www.fluxim.com.

[5] Schmidt, T. D., Jäger, L., Noguchi, Y., Ishii, H. and Brütting, W., "Analyzing degradation effects of organic lightemitting diodes via transient optical and electrical measurements” J. of Appl. Phys., 117, 215502. (2015)

[6] Dibb, G. F., Muth, M. A., Kirchartz, T., Engmann, S., Hoppe, H., Gobsch, G., Thelakkat, M., Blouin, N., Tierney, S., Carrasco-Orozco, M., Durrant, J. R. and Nelson, J., "Influence of doping on charge carrier collection in normal and inverted geometry polymer:fullerene solar cells” Scientific Reports, 3, 3335. (2013)

[7] Bloom, F., Langguth, O., Canzler,T., "Enabling dark injection SCLC to characterize trapping in OLED chrge transport layers” IDW Tech. Digest., 876-877, (2013)

[8] Nguyen, N. D., Schmeits, M. and Loebl, H. P., "Determination of charge-carrier transport in organic devices by admittance spectroscopy: Application to hole mobility in $\alpha$-NPD” Phys. Rev. B, 75, 075307-1, (2007).

[9] Knapp, E. and Ruhstaller,B. , "Analysis of negative capacitance and self-heating in organic semiconductor devices” J. Appl. Phys. 117 (13) 135501, (2015)

[10] Knapp, E. and Ruhstaller, B., "Numerical analysis of steady-state and transient charge transport in organic semiconductor devices” Optical and quantum electronics 42(11-13), 667-677. (2011)

[11] Ito, E., Washizu, Y., Hayashi, N., Ishii, H., Matsuie, N., Tsuboi,Y. Ouchi, K., Harima, Y., Yamashita K. and. Seki, K., "Spontaneous buildup of giant surface potential by vacuum deposition of Alq3 and its removal by visible light irradiation”, J. of Appl. Phys., 92, 7306-7310. (2002)

[12] Altazin, S., Clerc, R., Gwoziecki, R., Boudinet, D., Ghibaudo, G., Pananakakis, G., Chartier, I. and Coppard, R., "Analytical modeling of the contact resistance in top gate/bottom contacts organic thin film transistors" Org. Elec. 12, 897 ( 2011).

[13] Miyamae, T., Takada, N. and Tsutsui, T., "Probing buried organic layers in organic light-emitting diodes under operation by electric-field-induced doubly resonant sum-frequency generation spectroscopy”, Appl. Phys. Lett., 101, 073304 ( 2012).

[14] OLED and OPV characterization platform PAIOS version 2.0 by Fluxim AG, www.fluxim.com.

[15] Noguchi, Y., Lim, H., Isoshima, T., Ito, E., Hara, M., Chin, W. W., Han, J. W., Kinjo, H., Ozawa, Y., Nakayma, Y. and Ishii, H., "Influence of the direction of spontaneous orientation polarization on the charge injection properties of organic light-emitting diodes”, Appl. Phys. Lett., 102 (20), 203306 (2013).

[16] Kinjo, H., Lim, H., Sato, T., Noguchi, Y., Nakayama, Y. and Ishii, H., "Significant relaxation of residual negative carrier in polar Alq3 film directly detected by high-sensitivity photoemission” App. Phys. Express, 9, 021601 (2016). 